\title{
Anterior Aesthetic rehabilitation in a patient with suspectedamelogenesis imperfecta (AI)
}

\author{
${ }^{1}$ Dr. Vaibhav Kelkar ${ }^{2}$ Dr. Pranshu ${ }^{3}$ Dr.UzmaNazir \\ ${ }^{1}$ Postgraduate Resident, Department of conservative \& Endodontic, Inderprastha Dental College and Hospital, Ghaziabad, U.P \\ ${ }^{2}$ Postgraduate Resident, Department of conservative \& Endodontic, Inderprastha Dental College and Hospital, Ghaziabad, U.P \\ ${ }^{3}$ Orthodontist and Sleep Medicine Specialist at Siestabraces by city Dental clinic, Bohri kadal. Srinagar, J.K
}

DOI: http://dx.doi.org/10.47937/SJCMDS.2021.1105

\section{ABSTRACT}

Amelogenesis imperfecta (AI) is a genetic condition affecting the enamel of teeth with no system ic involvement. In this condition, patients can present changes in thickness, colour and shape of enamel, which leads to compromising the aesthetics and appearance of the patient along with impaored masticatory functions. Various treatment modalities are available for rehabilitation of such patients, like prosthetic procedures. This report shows a suspected case of AI and describes the clinical procedures of anterior aesthetic and functional rehabilitation of a young adult patient with involving the use of direct resin composite restorations and the use of all ceramic prosthesis. The overall result is remarkably different from the original appearance is a huge boost to the moral confidence of the patient.

Keywords: All ceramic, crowns, composite, veneer, direct veneer, Endodontics, RCT.

\section{Introduction}

Amelogenesis imperfecta (AI) can be defined as a group of rare conditions that are genetic and where the outer layer of the teeth (i.e. enamel) fails to properly develop. Patients with amelogenesis imperfecta usually have yellow to brown coloured teeth that are of smaller size and very prone to damage and breakage. (1) It affects the quality and quantity of dental enamel without any systemic involvement. It may affect some or all teeth of the permanent and/or deciduous dentition. The first classification of AI was published in 1945 by Weinmann. He divided AI into 2 basic groups: hypoplastic and hypocalcified. (2) Following this, a further classification by Witkop came in 1988 that divided the AI into four main groups and various subgroups (3):

Type I: Hypoplastic

Type II: Hypomaturation
Type III: Hypocalcified autosomal genetic trait

Type IV: Mixed Hypomaturation and Hypoplastic combined with Taurodontism

The hypoplastic (HP) AI and its subtypes show teeth with rough surfaces that are due to abnormalities in the formation of enamel and may also include agenesis of enamel. Many alterations combined with different genetic traits may be there. A normal radiographic contrast between enamel and dentin is evident. However, the thickness of enamel is significantly reduced. The defects have a deficiency in the quantity of enamel, which can be observed as pits, lines, grooves or thin enamel (3).

The hypomaturated (HM) AI shows alterations in enamel with a normal thickness but having a softer consistency. The enamel in such patients has a discoloured, mottled appearance and easily chips away. The radiocontrast of enamel in this type is similar to that of dentin. Hypomaturation type characteristically has enamel that appearing chalky and opaque (3). In the hypocalcified (HC) type, the enamel is malformed but of normal thickness. However, it is discoloured orange-yellow and generally the matrix is poorly calcified. This poor calcification results in a lower radiopacity compared to dentin. The hypocalcified varieties have alterations in enamel mineralization. The enamel appears discoloured and soft and can be easily removed (3).

The last type is shows the presence of an alteration of enamel combined with taurodontism. The enamel, which is malformed, when viewed histologically, shows a mixed appearance of hypomaturated and hypocalcified types of enamel with a radiocontrast which is similar to or slightly lower than that of dentin (3).

Treatment of patients with AI requires special care. The patients, especially the adolescents, teenagers and young adults are sometimes psychologically affected due to the unaesthetic appearance of their teeth, and aesthetic improvement can sometimes psychologically affected due to the unaesthetic appearance of their teeth, and aesthetic improvement can 
positively affect their self-confidence and behaviour. Below is a reported case of aesthetic management and rehabilitation of a young adult patient with AI using full coverage all ceramic crowns and with direct resin composite veneer restorations.

\section{CASE REPORT}

A 24-year old patient reported to the department of Conservative Dentistry and Endodontics with a chief complaint of unpleasant appearance of his teeth due to brownish-yellow discoloration on all the surfaces of his teeth. There was no pain or sensitivity associated with any of the teeth. There was no associated medical history. However, regarding the family history, patient informed that his father and his grandfather also suffered from discoloration of teeth which was similar in appearance to his own teeth. Intra oral examination revealed areas of heavily opaque white deposits on all his teeth along with varying degree of discoloration ranging from dark brown-to-light brown-to-yellow (Fig. 1 and Fig. 2) Moreover, there was also presence of generalized spacing between the teeth in his anterior region. Patient had a class I molar relation with a normal overjet and overbite. No decay or caries was seen in any teeth except for deep occluso-proximal caries with respect to tooth number 36 and 46 (Fig.3). The patient's chief complaint was the appearance of his teeth, i.e., he wished to get rid of discolorations on his front teeth. He wanted complete rehabilitation of his anterior teeth.

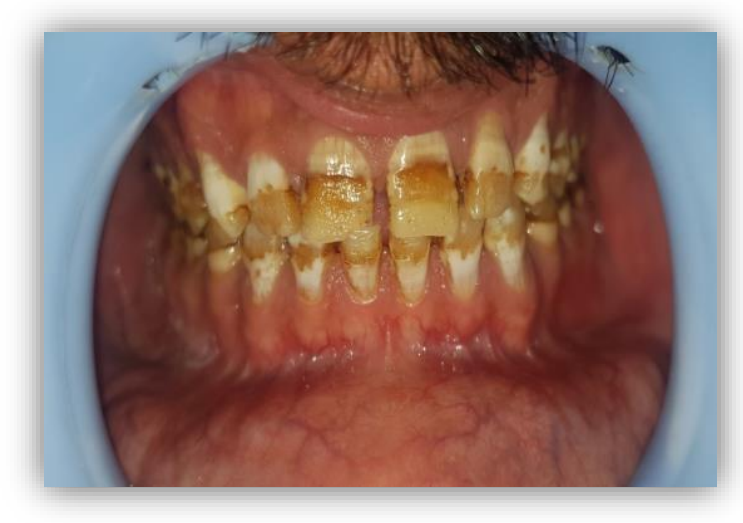

Fig. 2

Preoperative intraoral images

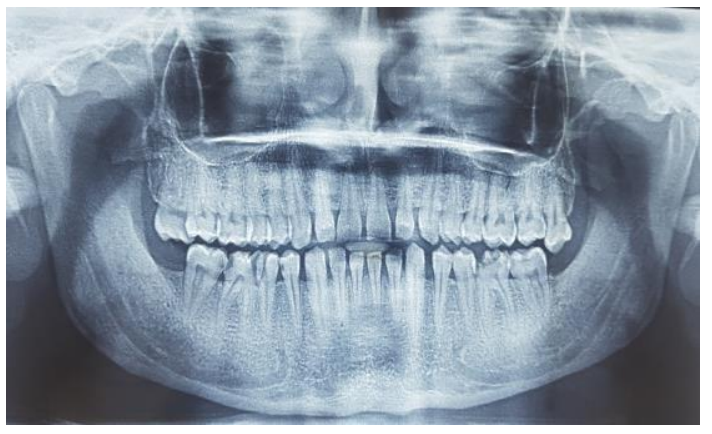

Fig. 3 Orthopantomogram of the patient
So, based on both the clinical as well as the radiographic findings and also taking into account the family history, a diagnosis of Amelogenesis Imperfecta Type II (hypomaturation type) was suspected and treatment plan was formulated as follows:

- Root canal treatment of anterior maxillary teeth followed by full coverage all ceramic crowns (teeth number 13, 12 11, 21, 22, 23)

- Direct composite veneers for mandibular anterior teeth (teeth numbers 33, 32, 31, 41, 42, 43).

The entire treatment plan was explained to the patient.

The patient's main desire was to achieve aesthetically pleasing results.

\section{PROCEDURE}

In the first two appointments, endodontic treatment was done for all maxillary anterior teeth. Single visit endodontic treatments were performed following standard protocol for all teeth. All the teeth were vital and hence were irrigated using $2.5 \%$ Sodium Hypochlorite for pulp devitalisation. For biomechanical preparation, \#30 0.06 taper Hero Shaper (MicroMega) File system was employed using manufacturers recommendation. Single cone obturation (along with accessary cones wherever required) was done for all teeth in the same appointment using Gutta Percha and Sealapex Sealer. (Fig. 4 - Fig 5)

\section{Fig. 4 Preoperative radiographs of maxillary anterior teeth.}

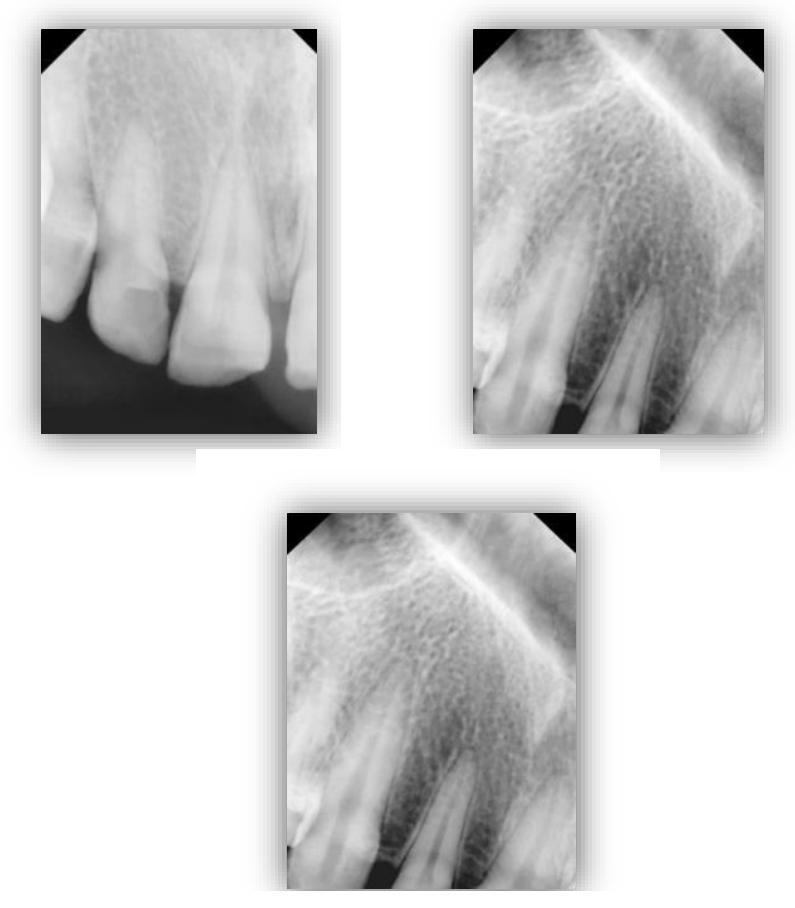



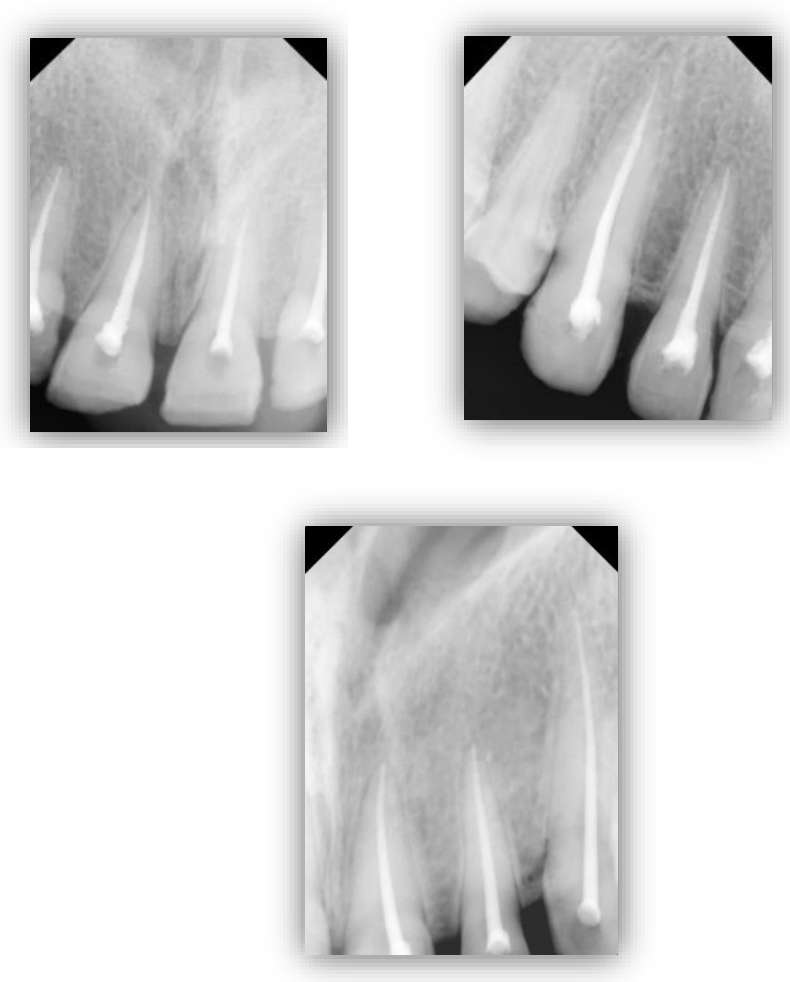

Fig. 5 Obturation radiographs of maxillary anterior teeth.

This was followed by crown preparation for all the anterior teeth for all ceramic restoration (Emax crowns) after a gap of 7 days (Fig. 6). Sufficient crown height was present in all teeth and hence no requirement of any crown lengthening procedures was there. A shoulder finish line was incorporated during the preparation. A Putty impression was made (Fig. 7) and gypsum Models were poured for the patient after the tooth preparation (Fig. 8a and b). Acrylic resin temporary crowns were prepared for the patient until his next appointment.

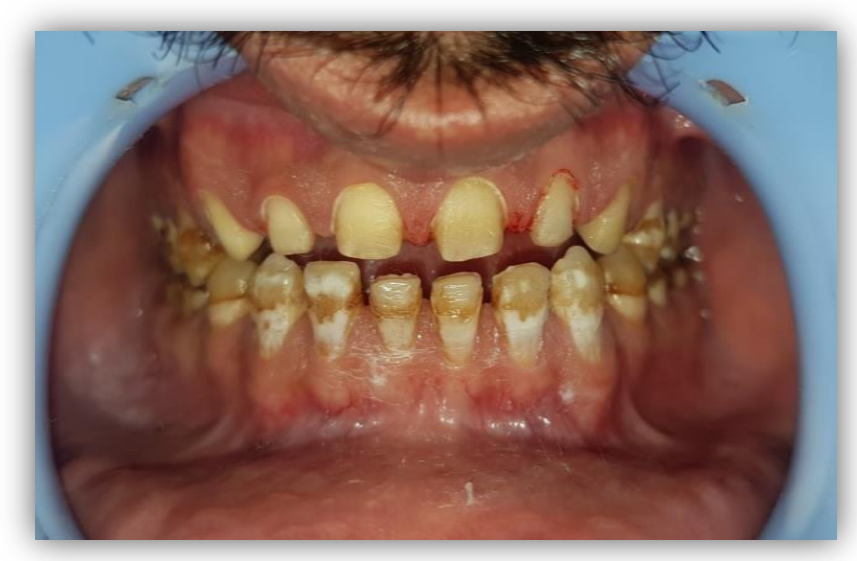

Fig. 6 Crown preparation - intraoral view

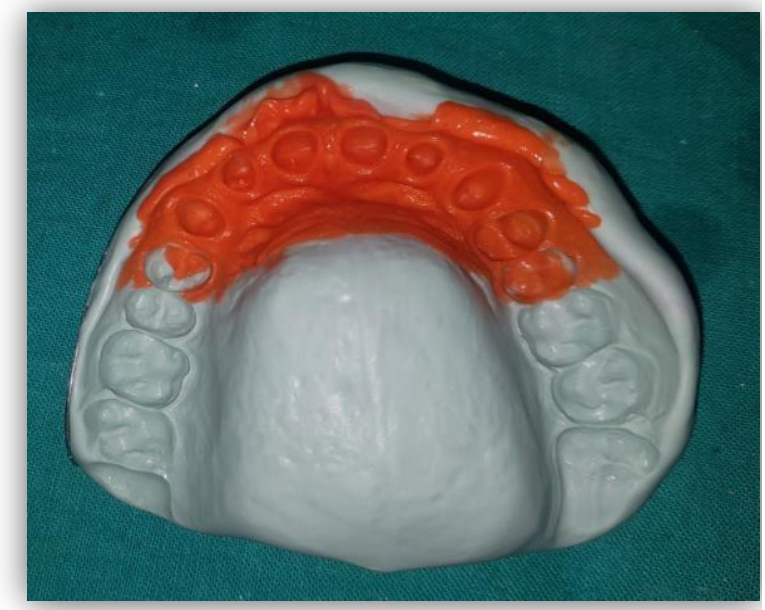

Fig 7 Putty Impression made

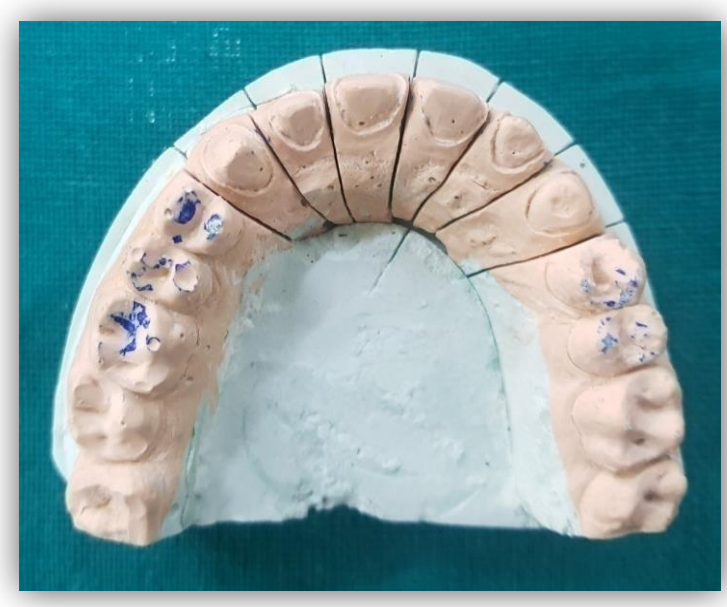

Fig. 8(a)

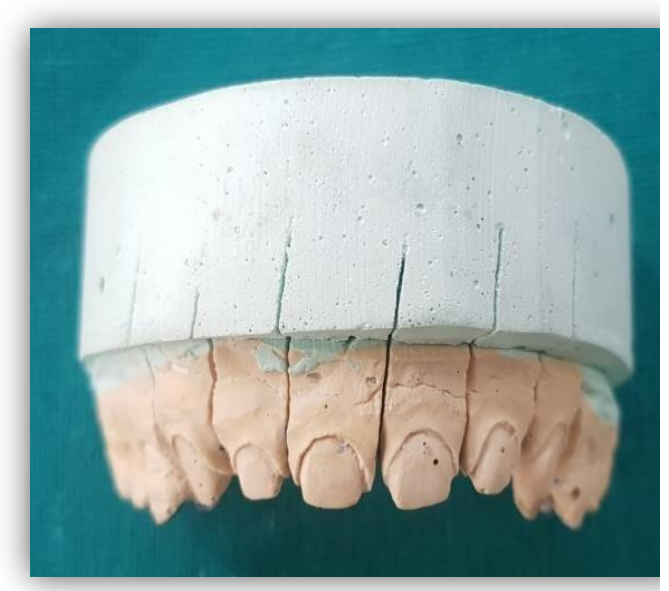

Fig. 8(b)

Gypsum Models poured 
In the next appointment preparation of the crowns was done in the anterior mandibular teeth for direct veneer restoration. 0.5-

$1.0 \mathrm{~mm}$ of tooth was prepared from the labial aspects as per the defects in the tooth structure, the depth of colour alteration and/or intensity of pigmentation. (4) (Fig. 9).

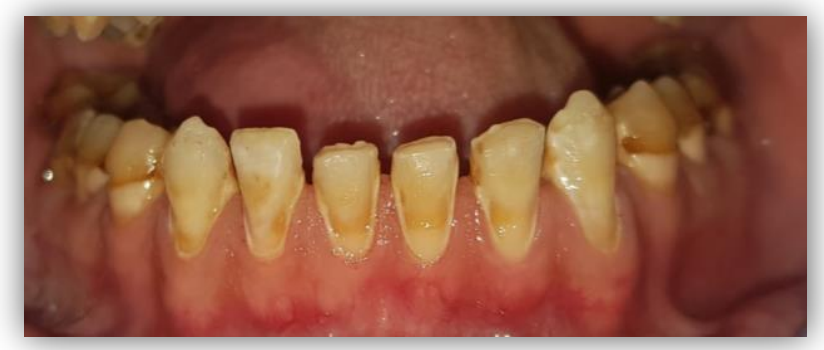

\section{Fig. 9 Direct veneer preparation on mandibular anterior teeth}

Direct composite restoration was done on mandibular anterior teeth using Te-Econom Plus (Ivoclar Vivadent) composite, after etching and bonding (Ivoclar Vivadent's Te Econom Bond 5th Generation which is a one bottle, one-layer adhesive; improves the bonding strength of the tooth to the composite with minimal marginal discoloration and sensitivity). (5) (Fig 10 - Fig 11).

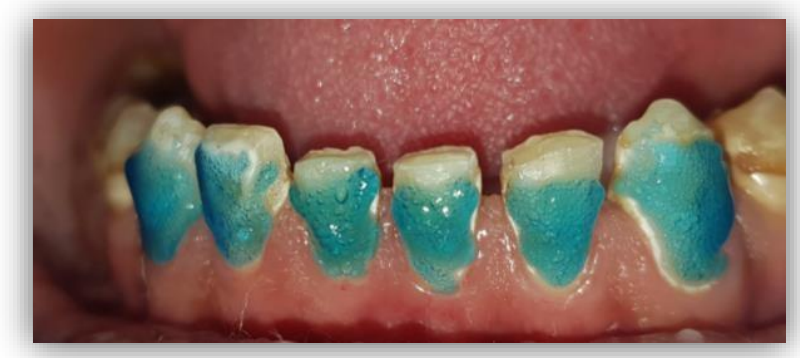

Fig. 10: Etching (Mandibular teeth)

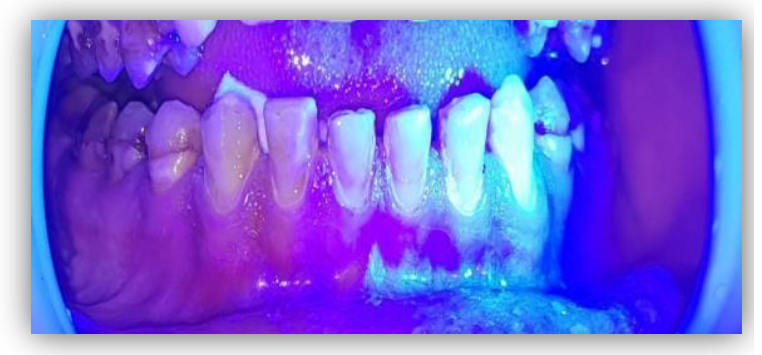

Fig 11: Bonding (Mandibular teeth)

After removing the temporary prostheses, maxillary teeth were etched and washed for 15 seconds followed by application of bonding agent (Ivoclar Vivadent's Te Econom Bond) (Fig 12 Fig 13).The all ceramic crowns were treated using Hydrofluoric acid (Ultradent) and washed for 15 seconds, followed by application of silane coupling agent for 10 seconds followed by gentle air drying. (Fig 14 - Fig 15).
Resin cement (Prevest), in a thin layer was applied on to the all ceramic crowns followed by placement of the crowns on the respective prepared teeth. After this, tag curing was done for 5 seconds from each surface. Any residual cement left on the toothprosthesis interface was removed using a sharp explorer. Finally, 30 seconds of curing was performed after the placement of each all ceramic crown.

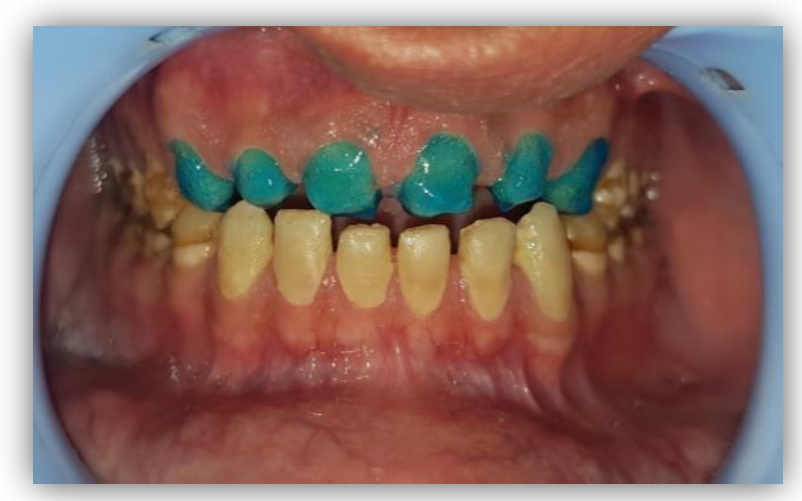

Fig. 12 Etching (Maxillary teeth)

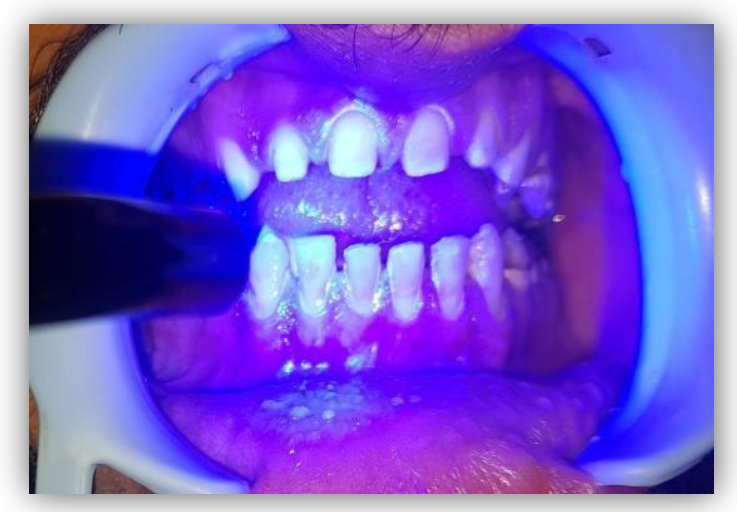

Fig 13: Bonding (Maxillary teeth)

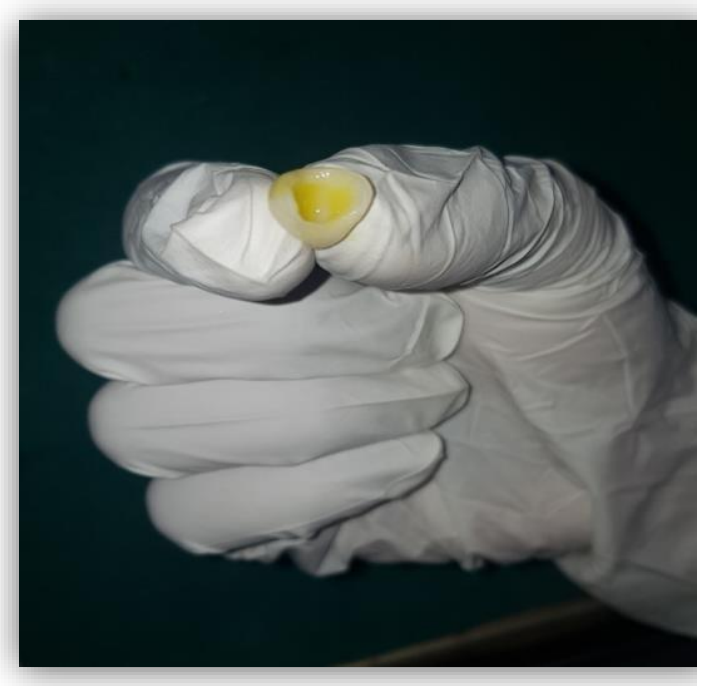

Fig 14 Applying Hydrofluoric acid 


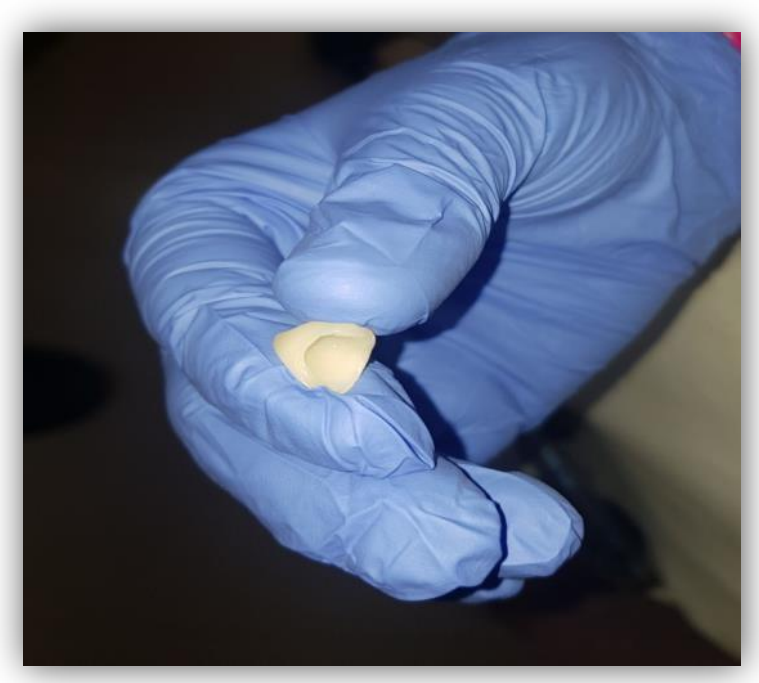

Fig 15: Applying Silane coupling agent

Final Post-operative image after the entire procedure can be seen as under (Fig. 16)

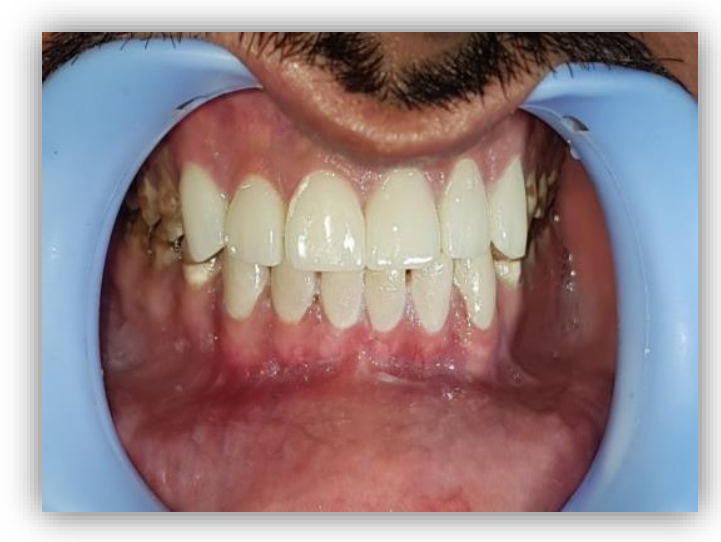

Fig 16: Final Post-Operative image

\section{Discussion:}

This was a suspected case of Amelogenesis Imperfect Type: Hypomaturation, treated by direct composite veneer and all ceramic crowns. Although the genetic examinations like gene mapping were not undertaken for the patient, still, owing to the clinical findings (like yellow to brownish discolouration of enamel, which was soft in consistency and having a somewhat chalky and opaque appearance), and comparison of radio contrast (similarity in radio density of enamel and dentin) (3), the suspected diagnosis was made of Hypomaturation type of AI.

Anitha $\mathrm{M}$ et al. (4) in a recent study and case series have described the types of AI along with the differentiating characteristics. Clinically, it might not be easy to differentiate among the various types of AI, but radio graphically, the type I, type II and type III are distinct. In the type I, the radio density difference between dentin and enamel is normal.
In type II, enamel is having similar radiodensity as dentin and it is usually not easy to make out radiographically any differentiating line between the two. In the third type, the enamel is having a reduced radiodensity as compared to dentin. In the present case as can be seen from the OPG and the RVGs there is not much radiodensity difference between dentin and and dentin. (4) Hence the diagnosis of Type II AI was made.

The treatment plan formulated and undertaken was all ceramic crowns for the anterior maxillary teeth and direct composite veneer restorations for the anterior mandibular teeth. This treatment plan was chosen based on patient's choice and his economic preferences. The patient decided to go for full coverage crowns for his maxillary teeth and not for his mandibular teeth. Instead, he opted for direct veneer composite restorations for his mandibular teeth for the simple reason of cost cutting. We could have gone for veneers (preferably direct) for the maxillary teeth also, as a more conservative approach. However, the patient walked into the department with a pre-decided treatment option for his upper teeth - he wanted "metal free full crowns for all his upper front teeth. Nonetheless, the end result was markedly different as compared to the pre-operative condition of the patient and this was a big positive change in the patient's life, behaviour and confidence. (Fig 17- Fig 18).

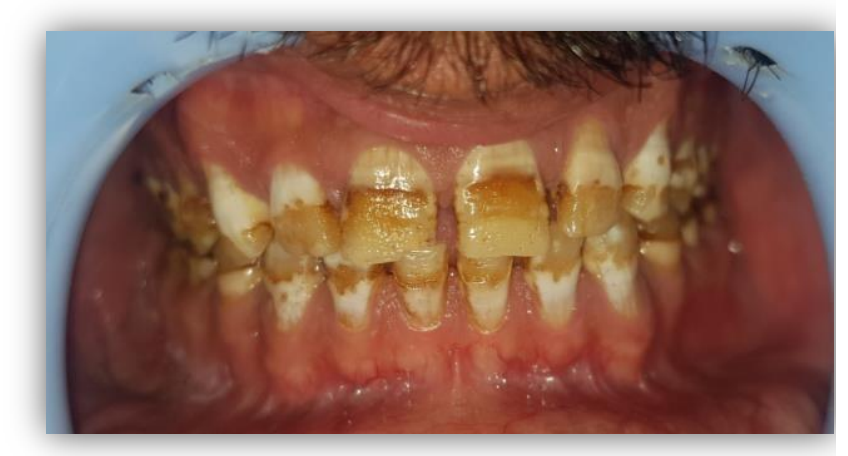

Fig. 17: Pre-operative intraoral image

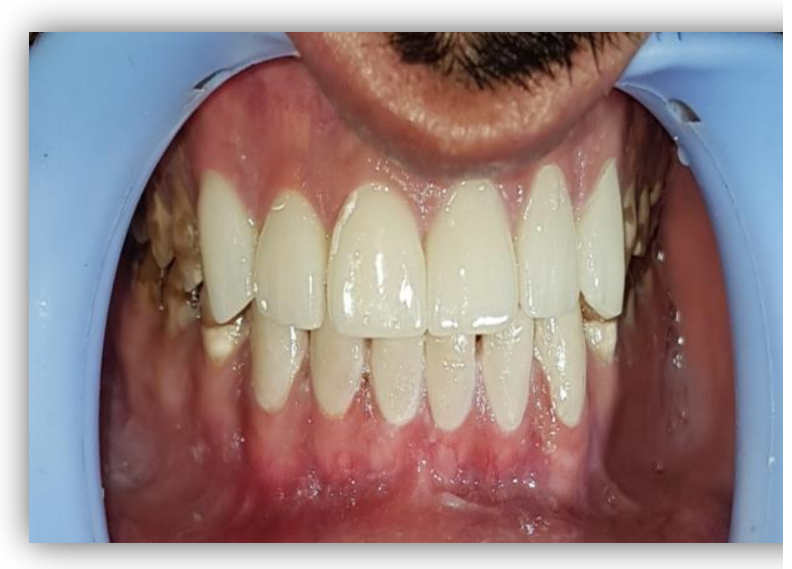

Fig. 18: Post-operative intraoral images 
The main challenge faced by the clinician while treating such patients is aesthetic correction because most of the times, that is the chief reason why the patients seek treatment in the first place. In the present case as well, there was no functional derangement or abnormality. The concern was chiefly aesthetic.

AI can be manifested by the mutations in ENAM, MMP20, AMELX, and in the FAM83H genes. These genes give instructions for making proteins essential for normal tooth development. Even though function of the protein produced by the FAM83H gene is unknown, it is also believed to have a role in the enamel formation. Most of these proteins have a role in the enamel formation, which is the calcium-rich, hard material that protects each tooth (6).

If there are mutations in any of these genes, it results in formation of altered protein or no protein at all. Hence, tooth enamel formed is thin or soft and can have an abnormal yellow or brown colour. Moreover, teeth formed having defective enamel are easily damaged and weak (7).

Various treatment options available in patients with AI depend on a number of factors like: (8)

- $\quad$ Patient's age,

- Patient motivation,

- Periodontal health of the patient,

- $\quad$ Endodontic status of the patient's tooth,

- $\quad$ Remaining tooth structure,

- Severity of the disorder,

- $\quad$ Socioeconomic status of the patient and

- Most importantly the patient's availability for treatment and cooperation

The restorative treatment in adolescents and onwards should always aim to restore the aesthetics, the health and the functional needs of the patient's teeth and prevent or delay the need for extraction and prosthetic management (9).

There are a number of Treatment Modalities that can be undertaken for the treatment of Amelogenesis Imperfect according to the severity and site: (10)

- $\quad$ Bleaching

- $\quad$ Direct composite Veneers

- $\quad$ Indirect Porcelain Veneers

- $\quad$ Full coverage gold crowns
- $\quad$ Full coverage All ceramic crowns

- $\quad$ Full coverage Porcelain fused to Metal crowns

In this case the prime concern of the patient was aesthetics. Hence, it was decided to go only for rehabilitation of anterior teeth. Moreover, the patient was only interested in getting full coverage crowns for his maxillary anterior teeth. He was satisfied with the direct composite restoration of his mandibular anterior teeth. However, the patient was warned against the potential future sensitivity of mandibular anterior teeth.

Strauch S. (10), in a recent review on the treatment considerations of AI, concluded that, the indirect restorations promise a much superior predictability and a better longevity compared to direct restorations, and this is particularly as a result of the poor-quality bonding of the adhesive, to enamel affected by AI. Moreover, the author also outlined that the type of AI seems to be insignificant for the restoration using crowns or Fixed dental prosthesis.

The Prognosis in this case was fair to good depending on various factors like the patient's age, the socio-economic condition of the patient and the awareness and ability to take care of his dentition. The patient was a healthy, 24-year old, adult patient who was having a decent socio-economic background and was well aware about oral hygiene. Still, he was warned about the cautious use of his anterior teeth especially his mandibular anterior teeth, the primary reason being that indirect restorations usually have a better prognosis compared to direct restorations. (6) Nonetheless, direct composite veneers have been employed as a treatment modality for $\mathrm{AI}$ in a 22-year old patient in one of the case reports. (11)

In nutshell, in this case, direct composite veneers were used due to their advantage of being a minimally invasive and relatively economically favourable approach in the anterior mandibular teeth. The maxillary anterior teeth were treated using all ceramic full crowns that promise a better longevity and remarkable aesthetics. The patient seemed satisfied with the end result. He was told to report to the dental office for regular follow-ups and also to maintain a good oral hygiene.

\section{Conclusion:}

This was a case report of the anterior management and rehabilitation of a young adult patient with AI treated using Full ceramic Crowns and Direct Composite Veneers. Direct composite veneers act as a promising minimally invasive technique in these types of cases. However, full ceramic prostheses offer far better longevity and superior aesthetics, and are also a viable treatment option available, especially in the anterior region. 


\section{References:}

1. Izgi A D et al, Amelogenesis Imperfect: Rehabilitation and Brainstorming on the Treatment Outcome after the First Year. Hindawi Publishing Corporation Case Reports in Dentistry 2015.

2. Weinmann JP, Svoboda JF, Woods RW. Hereditary disturbances of enamel formation and calcification. J Am Dent Assoc. 1945; 32:397-418.

3. Witkop CJ. Amelogenesis imperfecta, dentinogenesis imperfect and dentin dysplasia revisited: problems in classification. J Oral Pathol. 1988; 17:547-53.

4. Anitha et al.; Amelogenesis Imperfect- 3 Cases. JAMPS, 17(4): 1-8, 2018; Article no. JAMPS. 39759

5. Jorge Perdigão Saulo Geraldeli, James S. Hodges. Total-etch versus self-etch adhesive: Effect on postoperative sensitivity. JADA, 134(12): 1621-1629, December 2003

6. Yamaguti PM, Acevedo AC, de Paula LM; Rehabilitation of an Adolescent with Autosomal Dominant Amelogenesis Imperfect:Case Report. Operative Dentistry, 2006, 31-2, 266-272

7. ghr.nlm.nih.gov/condition/amelogenesis-imperfect(January 2020).

8. Martin M. I. Sabandal1 Edgar Schafer1. Amelogenesis imperfecta: review of diagnostic findings and treatment concepts Odontology (2016) 104:245-256

9. Geetima Khanna, Muhammad Nishad Thyath, Vikram Khanna, Meha Sharma. Amelogenesis imperfecta- A case report. J of Adv Med and Den Sci Research. 2015; 3(2)

10. Susanne Strauch, Dr. med. dent \& Sebastian Hahnel, Prof. Dr. med. Dent. Restorative Treatment in Patients with Amelogenesis Imperfecta: A Review. Journal of Prosthodontics 27 (2018) 618623

11. Tikku et al.; ARRB, 19(5): 1-4, 2017; Article no.ARRB.37740.

Cite This Article As: Kelkar V, Pranshu, Nazir U.

Anterior Aesthetic rehabilitation in a patient with

suspected amelogenesis imperfecta (AI). SJCMDS

2021; 1(1): 30-36 